\title{
Fading Statistics in Communications - a Random Matrix Approach
}

\author{
J.-H. Yeh, E. Ott, T.M. Antonsen And S.M. Anlage \\ University of Maryland, College Park, MD 20742, USA
}

\begin{abstract}
Fading is the time-dependent variations in signal strength measured at a receiver, due to temporally evolving multipath scattering and interference. In our previous work we introduced a statistical fading model for the time-reversal invariant case by combining the predictions of random matrix theory with the random coupling model that includes system-specific properties such as the radiation impedance of the ports and short-orbit effects. In the high-loss limit this random matrix theory model reduced to the most common fading models in the wireless communication field. In this paper we discuss the theoretical model in more detail and extend it to the case of broken time-reversal invariance.
\end{abstract}

PACS: $03.65 . \mathrm{Sq}$, 05.45.Mt, 42.25.Dd, 78.20.Bh

\section{Introduction}

The study of random matrix theory (RMT) is a highly developed field, and applications of RMT have been found in many different scientific and engineering disciplines [1]. The field was initiated by the work of Wigner $[2-5]$ concerning the statistics of the energy levels of large nuclei. Large nuclei are examples of complicated systems with extreme sensitivity to small changes, and therefore the exact solution is either inaccessible or may not be useful for predicting the properties of another similar system. To address this problem Wigner replaced the complicated Hamiltonian matrix $H$ of the system with a random matrix from a suitable ensemble. The statistical properties of the eigenvalues and eigenfunctions of these random matrices were found to be in agreement with those of real nuclei. Other researchers have extended the random matrix approach to other complicated systems in different fields, such as quantum systems [6, 7], wave scattering [8], acoustic waves [9], quantum dots and mesoscopic systems [10-12], and microwave cavities [13, 14].

This research encompasses an active field called "wave chaos" that utilizes a statistical approach to understanding the wave properties of complex systems. Classical chaos is characterized by the fact that small differences in the initial conditions of a dynamical system grow exponentially in time $[15,16]$. Although the wave equations in these systems are not chaotic, if the wavelength is very short compared to the typical length scale of the system, the so-called "semi-classical limit", the wave behavior can be well approximated by ray equations. Since the ray equations are a nonlinear Hamiltonian system, ray trajectories can be chaotic $[17,18]$. Wave chaotic systems are expected to show universal statistical properties, as predicted by RMT [19].
In this paper we study the application of the random matrix approach to fading statistics [20]. Consider wave propagation between a source and a receiver through a complex scattering environment. Fading is the time-dependent variation in the received signal amplitude as the scattering environment changes and evolves with time [21]. A common example is the night-time variation of AM radio signal reception in the presence of ray bounces off a time varying ionosphere. Another common observation of fading is experienced by radio listeners in automobiles moving among vehicles and buildings in an urban environment. Fading occurs because waves propagating via multiple paths or around obstacles interfere when they arrive at a receiver. Although fading exists in all types of wave propagation, such as electromagnetic waves, acoustic waves, and quantum waves, it has been most extensively studied in the wireless communication field [21-25]. Researchers have applied RMT in wireless communication [26] and in analyzing the information capacity of fading channels [27-30], but until recently, RMT has not been directly applied to the fading phenomenon itself [20].

In the wireless communication field, many statistical fading models are utilized to describe the fading phenomenon because practical wave propagating systems are generally complicated, and a precise mathematical description of the fading phenomenon is either unknown or too complex for tractable analysis [21]. Researchers have empirically created statistical models for fading channels, such as the Rayleigh fading model and the Rice fading model, that depend on the particular propagation environment and the underlying communication scenario [21]. Different fitting parameters are used in different models, for example, the Rayleigh fading model applies a one-parameter Rayleigh distribution to model the fading am- 
plitude in an environment where there is no line-of-sight (LOS) path between the transmitter and the receiver, such as mobile wireless systems in a metropolitan area [21-24]. The Rice fading model, on the other hand, applies a two-parameter distribution to model situations with a strong LOS path $[21,25]$.

In a previous paper we introduced a wave chaotic model of fading based on the combination of random matrix theory in the time-reversal invariant (TRI) case and the random coupling model [20]. We demonstrated that this new fading model is more complete than the Rayleigh and Rice fading models, which are recovered by our model in the high-loss limit. In this paper we present a thorough derivation of the new RMT fading model and extend the model to the time-reversal invariance broken (TRIB) case.

\section{Theory}

To model the fading amplitude, we use the scattering matrix $S$ that describes a linear relationship between the input and the output voltage waves on a network. We consider the $2 \times 2 S$ matrix, where the two ports of the network system correspond to the transmitter and the receiver. The complicated scattering system is modeled by the scattering matrix. The magnitude of the matrix element $\left|S_{21}\right|$ therefore corresponds to the fading amplitude. To apply the random matrix approach, we start with an RMT description of the $2 \times 2$ scattering matrix $s_{\text {rmt }}$ of a wave chaotic system, based on Brouwer and Beenakker's work [10]. This scattering matrix $s_{\text {rmt }}$ does not have any system specific information in it and is totally ergodic. We will introduce and include the system specific features later. The RMT form of the scattering matrix can be written as [10]:

$$
s_{\mathrm{rmt}}=U_{1}\left[\begin{array}{cc}
\sqrt{1-T_{1}} & 0 \\
0 & \sqrt{1-T_{2}}
\end{array}\right] U_{2} .
$$

In this equation $U_{1}$ and $U_{2}$ are two $2 \times 2$ random unitary matrices that can be written in the form [31]:

$$
U=\mathrm{e}^{\mathrm{i} \beta}\left[\begin{array}{cc}
\cos \theta \mathrm{e}^{\mathrm{i} \psi} & \sin \theta \mathrm{e}^{\mathrm{i} \varphi} \\
-\sin \theta \mathrm{e}^{-\mathrm{i} \varphi} & \cos \theta \mathrm{e}^{-\mathrm{i} \psi}
\end{array}\right],
$$

where $\beta, \psi$, and $\varphi$ are independent random variables uniformly distributing from 0 to $2 \pi$, and $\theta=\arcsin (\sqrt{\xi})$, where $\xi$ is a random variable uniformly distributing from 0 to 1 . In the time-reversal invariant case, $U_{2}$ is the transpose of $U_{1}\left(U_{2}=U_{1}^{\mathrm{T}}\right)$ [10], which in the case of broken time reversal symmetry $U_{1}$ and $U_{2}$ are independent (i.e., $\left(\beta_{1}, \psi_{1}, \varphi_{1}, \theta_{1}\right)$ and $\left(\beta_{2}, \psi_{2}, \varphi_{2}, \theta_{2}\right)$ are independent). Also, the quantities $T_{1}$ and $T_{2}$ in Eq. (1) are two random variables $\left(0 \leq T_{1} \leq 1\right.$ and $\left.0 \leq T_{2} \leq 1\right)$ whose joint distribution function depends on a scalar parameter $\gamma$, referred to as the "dephasing rate" [10]:

$$
P\left(T_{1}, T_{2} ; \gamma\right)=\frac{1}{8} \frac{\left|T_{1}-T_{2}\right|}{T_{1}^{4} T_{2}^{4}} \exp \left(-\frac{\gamma}{2}\left(\frac{1}{T_{1}}+\frac{1}{T_{2}}\right)\right)
$$

$$
\begin{aligned}
& \times\left[\gamma^{2}\left(2-2 \mathrm{e}^{\gamma}+\gamma+\gamma \mathrm{e}^{\gamma}\right)-\gamma\left(T_{1}+T_{2}\right)\left(6-6 \mathrm{e}^{\gamma}\right.\right. \\
& \left.+4 \gamma+2 \gamma \mathrm{e}^{\gamma}+\gamma^{2}\right)+T_{1} T_{2}\left(24-24 \mathrm{e}^{\gamma}+18 \gamma\right. \\
& \left.\left.+6 \gamma \mathrm{e}^{\gamma}+6 \gamma^{2}+\gamma^{3}\right)\right]
\end{aligned}
$$

From Eqs. (1) and (2), the fluctuating fading amplitude is

$$
\begin{aligned}
& \left|s_{\mathrm{rmt}, 21}\right|= \\
& \quad \sqrt{\xi(1-\xi)\left[2-T_{1}-T_{2}-2 \cos \phi \sqrt{\left(1-T_{1}\right)\left(1-T_{2}\right)}\right]},
\end{aligned}
$$

where $\phi=2(\psi-\varphi)$. Hemmady et al. [14, 32-36] found that the dephasing rate $\gamma$ in Eq. (3) can be related to the loss parameter $\alpha$ of the corresponding closed system as $\gamma=4 \pi \alpha$, where $\alpha \equiv f /(2 Q \Delta f), f$ is the frequency, $Q$ is the typical quality factor, and $\Delta f$ is the mean spacing of the adjacent eigenfrequencies. For an open fading system, we consider an equivalent closed system in which uniform absorption accounts for wave energy lost from the system, and we assume that we can define an equivalent loss parameter $\alpha$ for the open system.

From Eq. (4) we can tune the loss parameter $\alpha$, which determines the joint distribution function $P\left(T_{1}, T_{2} ; \gamma\right)$ (Eq. (3)), to generate different probability distribution functions of the fading amplitude [20]. In some special cases we are able to derive the analytical form of the distribution of the fading amplitude $P\left(\left|s_{\mathrm{rmt}, 21}\right|\right)$. For a lossless system $(\alpha=0)$, we have $T_{1}=T_{2}=0$ and

$$
\left|s_{\mathrm{rmt}, 21}\right|=\sqrt{2 \xi(1-\xi)(1-\cos \phi)} .
$$

By computing the $n$-th moment $\left\langle\left|s_{\text {rmt }, 21}\right|^{n}\right\rangle$, we find that the distribution of the fading amplitude is uniform for $0 \leq\left|s_{\text {rmt }, 21}\right| \leq 1$. For high loss systems $(\gamma \gg 1)$, the dominant term in Eq. (3) is $\gamma^{3} \mathrm{e}^{\gamma}$. Because of $0 \leq T_{1} \leq 1$ and $0 \leq T_{2} \leq 1$, the distribution $P\left(T_{1}, T_{2}\right)$ is negligible except when $T_{1} \rightarrow 1$ and $T_{2} \rightarrow 1$. Therefore, we use the approximation $1 / T_{1} \simeq 2-T_{1}$ and $1 / T_{2} \simeq 2-T_{2}$ in the exponential function in Eq. (3), and we keep only the dominant term. The joint distribution function $P\left(T_{1}, T_{2}\right)$ becomes

$$
\begin{aligned}
& P\left(T_{1}, T_{2} ; \gamma \gg 1\right)=\frac{\gamma^{3}}{8}\left|T_{1}-T_{2}\right| \\
& \quad \times \exp \left(-\frac{\gamma}{2}\left(2-T_{1}-T_{2}\right)\right) .
\end{aligned}
$$

With this joint distribution (Eq. (6)) and the formula of $\left|s_{\mathrm{rmt}, 21}\right|(\mathrm{Eq}$. (4)), we derive the $n$-th moment of the high-loss-limit distribution of $\left|s_{\mathrm{rmt}, 21}\right|$,

$$
\begin{aligned}
& \left\langle\left|s_{\mathrm{rmt}, 21}\right|^{n}\right\rangle=\int_{0}^{1} \mathrm{~d} \xi \int_{0}^{2 \pi} \frac{d \theta}{2 \pi} \int_{0}^{1} \mathrm{~d} T_{1} \\
& \quad \times \int_{0}^{1} \mathrm{~d} T_{2}\left|s_{\mathrm{rmt}, 21}\right|^{n} P\left(T_{1}, T_{2} ; \gamma\right)=\gamma^{\frac{-n}{2}} \Gamma\left(\frac{n}{2}+1\right) .
\end{aligned}
$$

This form of the $n$-th moment gives the distribution of $\left|s_{\text {rmt }, 21}\right|$ as 


$$
P\left(x=\left|s_{\mathrm{rmt}, 21}\right| ; \alpha\right)=8 \pi \alpha x \exp \left(-4 \pi \alpha x^{2}\right),
$$

which is identical to the Rayleigh distribution $P(x ; \sigma)=$ $\left(x / \sigma^{2}\right) \exp \left(-x^{2} /\left(2 \sigma^{2}\right)\right)$ with the relation

$$
\alpha=\frac{1}{8 \pi \sigma^{2}},
$$

valid for $\alpha \gg 1$.

For the TRIB case [10], we have a different joint distribution function $\widetilde{P}\left(T_{1}, T_{2}\right)$, and $U_{1}$ and $U_{2}$ in Eq. (1) are now independent random unitary matrices with independent random variables $\psi_{1}, \varphi_{1}, \beta_{1}, \psi_{2}, \varphi_{2}$, and $\beta_{2}$, which are all uniformly distributed from 0 to $2 \pi$. $\theta_{1}=$ $\arcsin \left(\sqrt{\xi_{1}}\right)$ and $\theta_{2}=\arcsin \left(\sqrt{\xi_{2}}\right)$ with independent random variables $\xi_{1}$ and $\xi_{2}$ uniformly distributing from 0 to 1 . The fluctuating fading amplitude for the TRIB case is

$$
\begin{aligned}
& \left|\widetilde{s}_{\mathrm{rmt}, 21}\right|^{2}=\xi_{1}\left(1-\xi_{2}\right)\left(1-T_{1}\right)+\xi_{2}\left(1-\xi_{1}\right)\left(1-T_{2}\right) \\
& \quad+2 \cos \widetilde{\phi} \sqrt{\xi_{1} \xi_{2}\left(1-\xi_{1}\right)\left(1-\xi_{2}\right)\left(1-T_{1}\right)\left(1-T_{2}\right)},
\end{aligned}
$$

where $\widetilde{\phi}=\psi_{1}+\psi_{2}+\varphi_{1}-\varphi_{2}$ has a uniform distribution on $[0,2 \pi)$.

We numerically generate $\left|\widetilde{s}_{\mathrm{rmt}, 21}\right|$ from Eq. (10) and plot the probability distributions $P\left(\left|\widetilde{s}_{\mathrm{rmt}, 21}\right|\right)$ with varying loss parameters in Fig. 1. The numerical results show that the distribution $P\left(\left|\widetilde{s}_{\mathrm{rmt}, 21}\right|\right)$ is a triangular distribution $P(x)=2 x$ for $0 \leq x=\left|\widetilde{s}_{\text {rmt }, 21}\right| \leq 1$ in the lossless case, different from the TRI case. However, the distribution goes to a Rayleigh distribution with the relation $\alpha=1 /\left(8 \pi \sigma^{2}\right)$ in the high loss limit, as with the time-reversal invariant case. In practice, time-reversal invariance for wave systems can be continuously broken $[18,37-39]$, so in the partially broken case the statistical properties would be in between the TRI case and the TRIB case.

The fading amplitude in Eq. (4) or (10) does not include the system-specific features yet. Therefore, in order to apply wave chaos theory to practical systems, we need to account for the non-chaotic features of the wave system. These non-chaotic features are specific to each wave scattering system. The random coupling model (RCM) is a method to combine the chaos properties and the non-chaotic system-specific features of a wave system in the impedance domain $[14,32-36,40-42]$. The impedance matrix $Z$ is the linear relationship between the port voltages and the port currents of a wave network, and it is related to the scattering matrix as $Z=Z_{0}(1+S) /(1-S)$, where $Z_{0}$ is a diagonal matrix with elements equal to the characteristic impedances of the transmission lines connected to the ports. In the ensemble wave-scattering system, the system-specific features include the radiation impedance $\left(Z_{\mathrm{rad}}\right)$ of the ports and short (major) trajectory orbits between the ports. The radiation impedance matrix $Z_{\text {rad }}$ quantifies the radiation and near-field characteristics of the ports.

The random coupling model [40-42] combines the system-specific features and the universal chaotic prop-

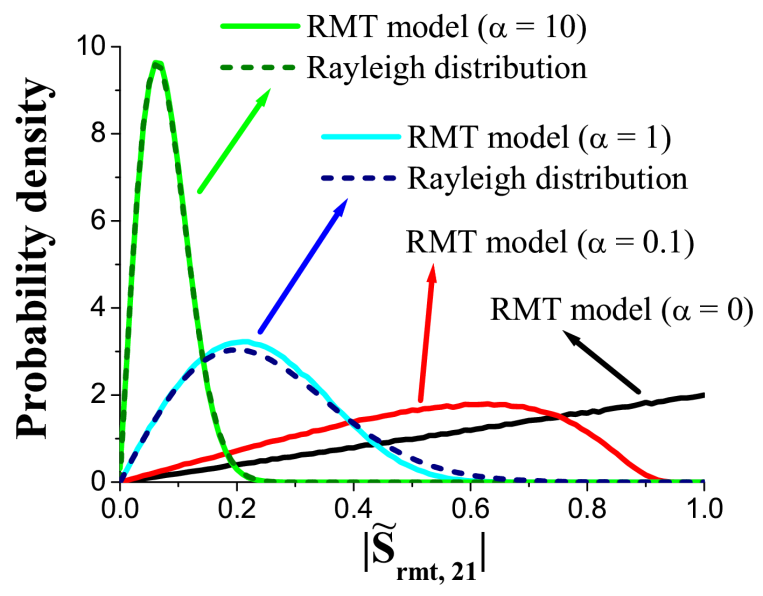

Fig. 1. The probability density functions of the fading amplitude $\left|\widetilde{s}_{\text {rmt }, 21}\right|$ for the TRIB case. Solid curves show the numerical results from the RMT model (Eq. (10)) with different loss parameters $(\alpha=0,0.1,1,10)$. For the higher loss cases $(\alpha=1$ and $\alpha=10)$, the corresponding Rayleigh distributions are shown as dashed curves.

erties as

$$
Z=\mathrm{i} X_{\mathrm{avg}}+R_{\mathrm{avg}}^{1 / 2} z_{\mathrm{rmt}} R_{\mathrm{avg}}^{1 / 2},
$$

where $z_{\mathrm{rmt}}$ is the RMT form of the impedance matrix computed by $z_{\mathrm{rmt}}=\left(1+s_{\mathrm{rmt}}\right) /\left(1-s_{\mathrm{rmt}}\right) . \quad R_{\mathrm{avg}}$ and $X_{\mathrm{avg}}$ are the resistance and the reactance parts of the ensemble-averaged impedance $Z_{\text {avg }}$ that encompasses the system-specific features. We then convert the impedance matrix back to the scattering matrix $S=\left(Z-Z_{0}\right) /\left(Z+Z_{0}\right)$ and have the complete statistical model for the measured fading amplitude $\left|S_{21}\right|$. The non-zero off-diagonal terms of $Z_{\text {avg }}$ bring $S_{21}$ a non-zero bias in the complex plane. We have shown that the bias corresponds to the $\nu$ parameter of the Rice distribution $P(x ; \sigma, \nu)=\left(x / \sigma^{2}\right) \exp \left(-\left(x^{2}+\nu^{2}\right) /\left(2 \sigma^{2}\right)\right) I_{0}\left(x \nu / \sigma^{2}\right)$ in the high-loss limit [20].

\section{Conclusion}

We start from the RMT prediction of the universal scattering parameter and combine it with the random coupling model to derive a statistical model for the fading parameter. This is a new application of wave chaos theory in the field of wireless communication, and this new model is more complete than traditional models. We also extend the theoretical model to the time-reversal invariance broken case. Because fading is a general phenomenon for all sorts of waves, our RMT fading model can, in addition to wireless communication field, also be applied to other wave scattering related applications. 


\section{Acknowledgments}

This work is funded by the ONR/Maryland AppEl Center Task A2 (contract No. N000140911190), the AFOSR under grant FA95500710049, and Center for Nanophysics and Advanced Materials (CNAM).

\section{References}

[1] M.L. Mehta, Random Matrices, 2nd ed., Academic Press, Boston 1991.

[2] E.P. Wigner, Ann. Math. 53, 36 (1951).

[3] E.P. Wigner, Ann. Math. 62, 548 (1955).

[4] E.P. Wigner, Ann. Math. 65, 203 (1957).

[5] E.P. Wigner, Ann. Math. 67, 325 (1958).

[6] F. Haake, Quantum Signatures of Chaos, Springer-Verlag, New York 1991.

[7] H.J. Stöckmann, Quantum Chaos, Cambridge University Press, Cambridge, England 1999.

[8] R.G. Newton, Scattering Theory of Waves and Particles, McGraw-Hill, New York 1966.

[9] V. Pagneux, A. Maurel, Phys. Rev. Lett. 86, 1199 (2001).

[10] P.W. Brouwer, C.W.J. Beenakker, Phys. Rev. B 55, 4695 (1997).

[11] Y. Alhassid, Rev. Mod. Phys. 75, 895 (2000).

[12] P.A. Mello, N. Kumar, Quantum Transport in Mesoscopic Systems, Oxford University Press, New York 2004.

[13] U. Kuhl, M. Martínez-Mares, R.A. Méndez-Sánchez, H.-J. Stöckmann, Phys. Rev. Lett. 94, 144101 (2005).

[14] S. Hemmady, Ph.D. thesis, University of Maryland, College Park 2006.

[15] M.C. Gutzwiller, Chaos in Classical and Quantum Mechanics, Springer-Verlag, New York 1990.

[16] E. Ott, Chaos in Dynamical Systems, 2nd ed., Cambridge University Press, New York 2002.

[17] H.-J. Stöckmann, J. Stein, Phys. Rev. Lett. 64, 2215 (1990).

[18] P. So, S.M. Anlage, E. Ott, R.N. Oerter, Phys. Rev. Lett. 74, 2662 (1995).

[19] O. Bohigas, M.J. Giannoni, C. Schmidt, Phys. Rev. Lett. 51, 1 (1984).

[20] J.-H. Yeh, E. Ott, T.M. Antonsen, S.M. Anlage, Phys. Rev. E 85, 015202(R) (2012).

[21] M. Simon, M.-S. Alouini, Digital Communication over Fading Channels, 2nd ed., Wiley-Interscience, Hoboken, NJ 2005.
[22] G.L. Stüber, Principles of Mobile Communications, Kluwer Academic Publishers, Norwell, MA 1996.

[23] G.J. Foschini, Bell Labs Technical J. 1, 41 (1996).

[24] O. Delangre, P. Doncker, M. Lienard, P. Degauque, C.R. Physique 11, 30 (2010).

[25] M. Nakagami, in: Statistical Methods in Radio Wave Propagation, Ed. W.C. Hoffman, Pergamon Press, New York 1960, p. 3.

[26] A.M. Tulino, S. Verdú, Foundat. Trends Commun. Inf. Theory 1, 1 (2004).

[27] A.L. Moustakas, H.U. Baranger, L. Balents, A.M. Sengupta, S.H. Simon, Science 287, 287 (2000).

[28] S.H. Simon, A.L. Moustakas, M. Stoytchev, H. Safar, Physics Today 54, 38 (2001).

[29] A.L. Moustakas, S.H. Simon, J. Phys. A, Math. Gen. 38, 10859 (2005).

[30] V.I. Morgenshtern, H. Bölcskei, IEEE Trans. Informat. Theory 53, 10 (2007).

[31] K. Zyczkowski, M. Kus, J. Phys. A, Math. Gen. 27, 4235 (1994).

[32] S. Hemmady, X. Zheng, E. Ott, T.M. Antonsen, S.M. Anlage, Phys. Rev. Lett. 94, 014102 (2005).

[33] S. Hemmady, X. Zheng, T.M. Antonsen, E. Ott, S.M. Anlage, Phys. Rev. E 71, 056215 (2005).

[34] S. Hemmady, X. Zheng, T.M. Antonsen, E. Ott, S.M. Anlage, Phys. Rev. E 74, 036213 (2006).

[35] S. Hemmady, X. Zheng, T.M. Antonsen, E. Ott, S.M. Anlage, Acta Phys. Pol. A 109, 65 (2006).

[36] S. Hemmady, J. Hart, X. Zheng, T.M. Antonsen, E. Ott, S.M. Anlage, Phys. Rev. B 74, 195326 (2006).

[37] U. Stoffregen, J. Stein, H.-J. Stöckmann, M. Kuś, F. Haake, Phys. Rev. Lett. 74, 14 (1995).

[38] S.-H. Chung, A. Gokirmak, D.-H. Wu, J.S.A. Bridgewater, E. Ott, T.M. Antonsen, S.M. Anlage, Phys. Rev. Lett. 85, 12 (2000).

[39] B. Dietz, T. Friedrich, H.L. Harney, M. Miski-Oglu, A. Richter, F. Schäfer, J. Verbaarschot, H.A. Weidenmüller, Phys. Rev. Lett. 103, 06410 (2009).

[40] J.A. Hart, T.M. Antonsen, E. Ott, Phys. Rev. E 80 , 041109 (2009).

[41] J.-H. Yeh, J.A. Hart, E. Bradshaw, T.M. Antonsen, E. Ott, S.M. Anlage, Phys. Rev. E 81, 025201(R) (2010).

[42] J.-H. Yeh, J.A. Hart, E. Bradshaw, T.M. Antonsen, E. Ott, S.M. Anlage, Phys. Rev. E 82, 041114 (2010). 